\title{
The Research of Power Dispatch Automation Monitoring System
}

\author{
Liting Wang ${ }^{\text {a }}$, Caifeng $\mathrm{Li}^{\mathrm{b}}{ }^{\mathrm{b}}$, Qihao Zhang, Yanping Li \\ State Grid Henan Electric Power Company Luoyang Power Supply Company, Luoyang 471900, \\ China \\ a1365597861@qq.com, b1015903240@qq.com
}

Keywords: Power dispatch, automation, monitoring.

\begin{abstract}
With the addition of automation system computers and communities equipment's', the security problem of computer's room appeared. In addition, because of hugeness of the automation system and the complex of the software, hardware structure, the exceptional problem maybe appeared in the system. For real-time watching the whole computer's room and the operation of the automation system, quick judgments and notify can be given in time, This article gives a thorough research of the surveillance alarm system for the automation of electric power system, and gives a whole design of the research, This article also detailed introduces the function of the system, the structure and technology realization of software and hardware, together we analyzed the sample.
\end{abstract}

\section{Introduction}

With the development of computer technology and expanding the grid size, the importance of automation professionals in the power system also greatly improved, while the processing power requirements of the automation system stronger, more demanding operational reliability. SCADA systems have become dispatcher implementation of the production command and control grid operation and grid operation indispensable tool for production of strong technical support. However, with computer automation systems and communication equipment of the rapid increase in the number, room safety issues emerged; and because the automation systems is very large, the software and hardware complex structure and function, the system itself may be a variety of unusual circumstances, such as: Automation system failures, network outages, data acquisition channel interrupts, system software anomalies.

If an exception occurs not in time, it will affect the normal operation of the automated system, leading to the dispatcher cannot be normal command and control, and severe cases may affect the safe operation of the entire power grid, causing huge economic losses.

Therefore, how to protect the security and stability of the automation system has become a problem of concern to the power system.

\section{The Problem of Electric Power Dispatch Automated Monitoring System to Solve}

With the development of computer technology, measurement and control technology, network technology and communication technology, the power system is moving towards automation, intelligent direction. Power system is also a strong proponent of "downsizing to improve efficiency ', thus making the" engine room unattended "has become a trend, and modern network, monitoring and control, communication means making the" engine room unattended "possible. To solve the SCADA system current problems, and "engine room unattended", it must has a real-time monitoring and alarm systems. The engine room unattended achieves real-time monitoring system must address the following key issues:

(1) Real-time information on the room environment to achieve real-time monitoring and automation systems, we must first get the room environment information and automation systems in real-time operating parameters. Among them, get room environment information by various sensors installed in the engine room and the signal acquisition means to achieve; and automated real-time operating parameters, will have to interface with the automation system approach to obtain. 
(2)After real-time monitoring information to obtain real-time information, it is need for real-time monitoring of these data, which is central to the system. Due to the need to monitor very large amount of information, matters very much need to be monitored, such information must be classified portfolio, be judged according to different logic algorithms. Among them, the common logic algorithm is: unbalanced trend data is not refreshed, the more limited data, data hopping, remote displacement and the like.

(3) real-time monitoring alarm information release system diagnostic fault information out of the system that currently exist, and how these fault information timely and accurate delivery to the relevant personnel in the hands, and to avoid failure information omission, misstatement, repeat broadcast become realized Difficulties of this system lies. At present, the commonly used means of publication alarms include: Audible alarm, marquee, fixed phone alarm, SMS alarm.

(4) Room information inquiry on the one hand, the staff room and automation systems need to understand the current operating situation, in order to make the appropriate adjustments and treatment; in addition, the staff of the system through the query historical statistics, you can learn automation equipment in operating conditions over time, to prepare for future maintenance work. At present, the commonly used query tools have: WEB query system (including real-time information, historical information, and video screen), telephone and on-site query language query system.

Monitoring objects (5) automation system network security room monitoring system is the most important real-time systems, with the highest demand for network security, system design should be safe in the first place, it is necessary to ensure the EMS / DMIS / TMR system is stable and reliable, and To prevent external intrusion via the network. Therefore, according to the provisions on network security and system security grid scheduling, automation equipment and room monitoring alarm system should be placed in the third district network security requirements

\section{The Function of Dispatching Automation Monitoring System}

These existing monitoring systems in the engine room monitoring environment has matured, but according to the current situation of power dispatching automation systems, environmental monitoring alone cannot meet the requirements, but the need for hardware and software equipment operation fully automated system intelligent monitoring system to monitor, power dispatching automation monitoring and warning system of the present study combines the latest multimedia technology, computer room environment, network, system, data of four monitoring, alarm, control system so that "data level alarm" through various anomalies that may arise EMS system to conduct a comprehensive analysis, using rigorous scientific alarm logic, making all kinds of anomalies are being monitored for operational monitoring automation system itself provides a complete solution.

\section{Data Collection.}

According to their characteristics automated monitoring alarm system designed with an industrial-grade processing unit as a monitoring and control host, with AC and DC hybrid sampling module, remote acquisition module, plus for measurement of CT, PT board, relay board, each monitor floor or area alone group screen, forming a collection with integrated unit data processing and transmission functions, each integrated unit can access 32 telemetry information, remote communication signals 48-point, 16-way remote control signal, integrated unit and monitoring between the front-end network systems approach to communication. Thus, a plurality of integrated front-end unit and the network constitutes a data collection network. In addition, it set aside port; data can be collected to be sent to the sub-station RTU mode dispatch center or other systems.

\section{Data interface.}

Because monitoring alarm system is designed for SCADA systems and design, in addition to the data collected to monitor, but also for dispatching automation system (EMS), electricity metering system (TMR), data integration systems, network security systems, scheduling data network systems, real-time channel receiver systems, scheduling, production information management system (DMIS), UPS power supply, air conditioning and other systems (equipment), process, data, status and other information can be monitored. Monitoring alarm system software through the interface with other 
systems to exchange information, the interface software is modular in design, in the form of the statute reflects the library, the library can store all the national standard, the power industry or IEC standard transmission protocol can also be programmed by the user the definition of the statute was added to the statute library. If the amount of information exchanged is not too large, you should try to use the serial port to communicate the way, to avoid damage to the closed node monitoring system and network security.

\section{Core monitoring and management.}

System provides a user-friendly maintenance interface, maintenance personnel can easily modify all the database information table information, system parameters, user permissions table, table and other responsible personnel information. Data monitoring is the core of the entire monitoring alarm system. It is responsible for the collection of environmental data room as well as a summary of the available data interface from SCADA system, analyzed according to pre-set the alarm logic, judgment, when the alarm condition is established, the system will generate an alarm message and alarm Information sent through the network to "multimedia alarm system", the release alarm information to relevant personnel from the "multimedia alarm system." Room environment monitoring and warning system is essential to monitor objects in the room or by installing local area temperature sense probe (or temperature and humidity transmitter), flood detectors, smoke detectors and other devices to monitor the room temperature (or humidity) flood, fire or other abnormal conditions. When the temperature beyond alarm set point, smoke, water displacement detector generates remote communication, intelligent alarm system will trigger the start. Monitored alarm system from the factory station channel state values EMS, TMR systems, such as special virtual remote signaling point, involved in intelligent alarm, when a station channel interrupts or the error rate is too high, the system will be released in the smart alarm abnormal channel signal.

\section{Multimedia Intelligent Alarm.}

Multimedia intelligent alarm monitoring subsystem is one of the important functions of the alarm system, but also the user to use the most direct function of monitoring and warning system for all abnormal or alarm information is timely to contact the user through it. With multimedia intelligent alarm subsystem, greatly reducing the labor intensity of maintenance personnel to ensure that the automation system to operate more efficiently, reduce accidents, reflecting the economic production management. Duty way multimedia smart alarm subsystem can be flexibly set: Duty is divided into "duty cycle" and "Date duty." System can be set up once the duty cycle table; you can also set the duty at a particular period (Day, holidays). Each alarm has different levels of user (client), namely sub-level user. When the smart alarm release warning information, the warning is priority paging levels parties of the information, and secondly secondary and tertiary users. When a class of alarm is coming, monitoring alarm system will notice all users.

\section{Telephone inquiry.}

Telephone inquiry system is mainly connected by a smart card to the program-controlled telephone voice network, the user can call room monitoring system telephone alarm query, the query engine room the latest environmental information, the latest alarm information. Enquiries by the police, the police responsible person can listen to the latest alarm information with voice playback, including fault location and fault phenomenon, as well as six alarm numbers for alarm acknowledgment.

\section{Summary}

In this paper, an automated system operation and management as the starting point, with SCADA computer room environment for the safe operation of the electric power dispatch automated monitoring alarm system was serious, in-depth research, and put forward a complete research programs. Operation and management mode operation of the system dispatching automation system marks the transition from staff on duty to "intelligent operation and management" in the true sense, so that operation scheduling automation systems, management level on a new level. 


\section{Reference}

[1] Sun L, Shen S. Design and Application of Wind Power Dispatch Automation System [J]. Wind Energy, 2012.

[2] Song J, Xiaobin L I, Yao J, et al. Design and Implementation of Multi-protocol [3] Communication Software Platform of Reservoir Automation Dispatch System [J]. Hydropower Automation \& Dam Monitoring, 2011.

[4] Cheng T. Reform of power dispatch and monitoring system in Yangzi [J]. Science \& Technology Information, 2011.

[5] Ma K M. Introduction to Power Dispatch SCADA System of Guangzhou-Shenzhen 10 kV Power Line [J]. Electric Power Automation Equipment, 2000.

[6] Liang M X. Picture Display Dispatch Strategy in Monitoring System [J]. Electric Power Automation Equipment, 2000. 